\title{
Comparison of Myometrial Invasion and Tumor Free Distance from Uterine Serosa in Endometrial Cancer
}

\author{
Ozlem Ozbilen $^{1}$, Derya Kilic Sakarya ${ }^{2 *}$, Incim Bezircioglu ${ }^{3}$, Burcu Kasap ${ }^{4}$, Hakan \\ Yetimalar $^{3}$, Seyran Yigit ${ }^{5}$
}

\begin{abstract}
Background: We aimed to investigate whether the tumor free distance (the distance between the uterine serosa and the tumor at its deepest point) is useful in surgical staging and in predicting prognosis. Materials and Methods: Data from patients who underwent complete surgical staging for endometrial cancer between January 2006 and June 2011 were reviewed retrospectively. All demographic findings, surgical stages, histological type and grade, myometrial invasion, lymphovascular space invasion as well as abdominal cytology, cervical, adnexal, and omental involvement, and lymph node metastasis were recorded. The relations between myometrial invasion and tumor free distance from uterine serosa with prognostic factors were investigated. Results: Seventy patients were included in the study. Sixty-four (91.5\%) had endometrioid type cancers and forty-four (62.9\%) were grade 1 . The deepest myometrial invasion was less than $1 / 2$ in 42 patients $(60 \%)$. In 18 patients $(25.8 \%)$ lymphovascular invasion was noted. Eight $(\mathbf{1 1 . 4 \% )}$ ) were found to have cervical involvement, five $\mathbf{( 7 . 1 \% )}$ had adnexal involvement and in 4 cases $(5.7 \%)$ the peritoneal washings included malignant cells. Four patients had pelvic and one para-aortic node metastasis. We recognized that an invasion of more than $1 / 2$ was correlated significantly with lymphovascular space involvement, histological grade, positive abdominal washing cytology, nodal and cervical involvement, but not with adnexal involvement. Tumor-free myometrial thickness was negative and statistically significant correlated with surgical stage, histological grade, lymphovascular space involvement, positive abdominal washing cytology, cervical and adnexal involvement. The importance of tumorfree myometrial thickness in determinating the lymphovascular space invasion was found to be highest in terms of sensitivity and specificity when crossing the ROC curve at 11 millimeters. Conclusions: Depth of myometrial invasion is more valuable for predicting lymph node metastasis than tumor-free myometrial thickness. The tumor-free myometrial thickness provides a better prediction for adnexal involvement.
\end{abstract}

Keywords: Endometrium cancer - myometrial invasion - tumor-free distance - cancer staging - prognosis

Asian Pac J Cancer Prev, 16 (2), 519-522

\section{Introduction}

Endometrial cancer is the most common gynecologic malignancy and the fourth most common cancer in women, comprising \%6 of female cancers in developed countries (Ferlay et al., 2010; Siegel et al., 2013). The management of endometrial cancer is done with and according to surgical staging. Aside from stage, depth of myometrial invasion is an important prognostic factor and essential for surgical staging and management (Vargas et al., 2014; Rahatli et al., 2014). It is a prognostic factor for extrauterine spread (Vargas et al., 2014; Rahatli et al., 2014). However establishing myometrial invasion (MI) of endometrial cancer can be difficult in cases with different patterns of MI or tumor growth and irregular endomyometrial junction due to leiomyomas and adenomyosis (Quick et al., 2012). In such patients, the tumor free distance (TFD) (the distance between the deepest point of invasion and the uterine serosa) may provide a better prediction and be an alternative measurement method (Kondalsamy-Chennaksavan et al., 2010).

It was our aim to investigate the value of the TFD from uterine serosa in endometrial adenocarcinoma in surgical staging and its effects on several prognostic factors. For this purpose, the depth of myometrial invasion (DOI), TFD and the presence of MI more than $1 / 2$ were analyzed and compared in the current study.

\section{Materials and Methods}

All data from patients which underwent complete surgical staging for endometrial cancer in Izmir Ataturk Training and Research Hospital between January 2006 and

${ }^{1}$ Department of Obstetrics and Gynecology, Giresun Maternity and Pediatric State Hospital, Giresun, ${ }^{2}$ Department of Obstetrics and Gynecology, Bozkir State Hospital, Konya, ${ }^{4}$ Department of Obstetrics and Gynecology, Mugla Sitki Kocman University School of Medicine, Mugla, ${ }^{3}$ Department of Obstetrics and Gynecology, ${ }^{5}$ Department of Pathology, Izmir Katip Celebi University Ataturk Training and Research Hospital, Izmir, Turkey*For correspondence: deryakilicsakarya@ gmail.com 
Ozlem Ozbilen et al

June 2011 were collected retrospectively from medical records. Complete surgical staging with hysterectomy, bilateral salpingo-oopherectomy, and lymph node sampling were done routinely in all cases. It is our strategy to perform pelvic/paraaortic lymph node (LN) sampling in histologically grade $1-2$ cases with $\mathrm{MI}<1 / 2$ and systematic pelvic/paraaortic LN dissection in more advanced cases. The DOI, TFD, presence of MI more than $1 / 2$, lymphovascular space involvement (LVSI), ascites cytology, cervical, adnexal, and omental involvement, as well as lymph node (LN) metastasis were recorded. The TFD and DOI measurements re-checked by a gynaecological pathologist retrospectively. The relations of the degree of MI, TFD and prognostic factors were investigated.

Statistical analysis was calculated using SPSS 15.0 for Windows (SPSS Inc, Chicago, III, USA) statistical software. Categorical variables were described using frequency distribution. For comparing categorical variables, Pearson and Fisher's chi-square tests were used. Pearson's and Spearman's coefficient for correlation was used for commenting the connections. $\mathrm{p}<0.05$ was accepted as the level of significance.

\section{Results}

The mean age of 70 patients enrolled in the study was $62.6 \pm 9.9$ and $93.4 \%$ of the patients were in the postmenopausal period. In 64 patients $(91.5 \%)$, the hystopathological diagnosis was endometrioid. Fourtyfour patients $(62.9 \%)$ were grade 1,15 patients $(21.4 \%)$ were grade 2,11 patients $(15.7 \%)$ were grade 3 . The deepest myometrial invasion was less than $1 / 2$ in 42 patients (60\%). When LN metastasis was investigated, pelvic $\mathrm{LN}$ was detected in 4 patients $(5.7 \%)$, and paraaortic LN metastasis was detected in one patient $(1.4 \%)$, respectively. In 18 patients (25.8\%) LVSI was found. Eight patients $(11.4 \%)$ were found to have cervical involvement. Five patients $(7.1 \%)$ had adnexal ivolvement and in 4 cases $(5.7 \%)$ the peritoneal washings included malignant cells. Clinical and hystopathological characteristics were summarized in Table 1.

The association between the presence of MI more than $1 / 2$ and nodal involvement, LVSI, grade, positive cytology, cervical and adnexal involvement was investigated. LN metastasis, LVSI, grade, cervical involvement and positive cytology were significantly associated with $\mathrm{MI} \geq 1 / 2$. The presence of adnexal involvement was not significantly associated with MI. The association between the presence of MI more than $1 / 2$ and the prognostic variables was showed in Table 2.

The association between quantitative measurements of the DOI and the prognostic variables was investigated and the results were shown in Table 3. LVSI, grade, cervical and adnexal involvement were significantly associated with the DOI. LN involvement and positive cytology were not associated with the DOI.

The relation of the TFD with the prognostic variables were pointed out in Table 4. TFD was negatively correlated with the grade, positive cytology, surgical stage, LVSI, cervical involvement and adnexal involvement $(\mathrm{p}<0.05)$.
But no significance was found for LN metastasis, hystological type and tumor size.

On hystopathological investigation, mean-median myometrial thickness value was $17.3 \mathrm{~mm}(5-32 \mathrm{~mm})$,

Table 1. Patient Characteristics

\begin{tabular}{|c|c|c|}
\hline & Patients' number (n) & $\%$ \\
\hline \multicolumn{3}{|l|}{ Histologic type } \\
\hline Endometrioid & 64 & 91.5 \\
\hline Serous & 3 & 4.3 \\
\hline Squamous & 1 & 1.4 \\
\hline Clear cell & 1 & 1.4 \\
\hline Mixt (endometrioid/musinous) & 1 & 1.4 \\
\hline \multicolumn{3}{|l|}{ Histologic grade } \\
\hline 1 & 44 & 62.9 \\
\hline 2 & 15 & 21.4 \\
\hline 3 & 11 & 15.7 \\
\hline \multicolumn{3}{|l|}{ Myometrial invasion } \\
\hline$<1 / 2$ & 42 & 60 \\
\hline$\geq 1 / 2$ & 28 & 40 \\
\hline \multicolumn{3}{|l|}{ Lymphovascular space invasion } \\
\hline positive & 18 & 25.7 \\
\hline negative & 52 & 74.3 \\
\hline \multicolumn{3}{|l|}{ Cervical involvement } \\
\hline positive & 8 & 11.4 \\
\hline negative & 62 & 88.6 \\
\hline \multicolumn{3}{|l|}{ Adnexal involvement } \\
\hline positive & 5 & 7.1 \\
\hline negative & 65 & 92.9 \\
\hline \multicolumn{3}{|l|}{ Lymph node metastases } \\
\hline pelvic & 4 & 5.7 \\
\hline paraaortic & 1 & 1.4 \\
\hline \multicolumn{3}{|l|}{ Myometrial thickness } \\
\hline $0-10 \mathrm{~mm}$ & 7 & 10.0 \\
\hline $11-20 \mathrm{~mm}$ & 47 & 67.2 \\
\hline $21-30 \mathrm{~mm}$ & 15 & 21.4 \\
\hline $31-40 \mathrm{~mm}$ & 1 & 1.4 \\
\hline \multicolumn{3}{|l|}{ Depth myometrial invasion } \\
\hline $0-5 \mathrm{~mm}$ & 34 & 48.6 \\
\hline $6-10 \mathrm{~mm}$ & 17 & 24.3 \\
\hline $11-15 \mathrm{~mm}$ & 10 & 14.3 \\
\hline $16-20 \mathrm{~mm}$ & 7 & 10.0 \\
\hline $21-25 \mathrm{~mm}$ & 1 & 1.4 \\
\hline $26-30 \mathrm{~mm}$ & - & - \\
\hline $31-35 \mathrm{~mm}$ & 1 & 1.4 \\
\hline \multicolumn{3}{|l|}{ Tumor-free distance from serosa } \\
\hline $0-5 \mathrm{~mm}$ & 22 & 31.4 \\
\hline $6-10 \mathrm{~mm}$ & 18 & 25.7 \\
\hline $11-15 \mathrm{~mm}$ & 11 & 15.8 \\
\hline $16-20 \mathrm{~mm}$ & 15 & 21.4 \\
\hline $21-25 \mathrm{~mm}$ & 4 & 5.7 \\
\hline \multicolumn{3}{|l|}{ Form of the tumor } \\
\hline Solid & 29 & 41.4 \\
\hline Superficial & 13 & 18.6 \\
\hline Fulling the whole cavity & 28 & 40.0 \\
\hline \multicolumn{3}{|l|}{ Cytology } \\
\hline Benign & 65 & 92.9 \\
\hline Malign & 4 & 5.7 \\
\hline \multicolumn{3}{|l|}{ Surgical stage } \\
\hline $1 \mathrm{~A}$ & 39 & 55.8 \\
\hline $1 \mathrm{~B}$ & 18 & 25.7 \\
\hline 2 & 3 & 4.3 \\
\hline $3 \mathrm{~A}$ & 1 & 1.4 \\
\hline $3 \mathrm{C} 1$ & 4 & 5.7 \\
\hline $3 \mathrm{C} 2$ & 1 & 1.4 \\
\hline 4 & 1 & 1.4 \\
\hline
\end{tabular}


Tumor Free Distance from Uterine Serosa is a Prognositc Factor for Endometrial Cancer

Table 2. The Effect of the Presence of more than 1/2 Myometrial Invasion on Prognostic Variables

\begin{tabular}{lcc}
\hline & Myometrial invasion & p value \\
\hline Histologic grade & $<1 / 2$ & $<0.0001$ \\
& $\geq 1 / 2$ & $<0.0001$ \\
Lymphovascular space invasion & $<1 / 2$ & 0.0324 \\
& $\geq 1 / 2$ & $<0.0001$ \\
Cervical involvement & $<1 / 2$ & 0.005 \\
& $\geq 1 / 2$ & $<0.0001$ \\
Adnexal involvement & $<1 / 2$ & 0.348 \\
& $\geq 1 / 2$ & 0.099 \\
Positive cytology & $<1 / 2$ & 0.0412 \\
Lymph node involvement & $\geq 1 / 2$ & $<0.0001$ \\
& $<1 / 2$ & 0.101 \\
& $\geq 1 / 2$ & 0.002 \\
\hline
\end{tabular}

Table 3. The Association of the Depth of Myometrial Invasion of the Tumor and Prognostic Variables

\begin{tabular}{lcc}
\hline & $\begin{array}{c}\text { Depth of myometrial invasion } \\
\text { rho value }\end{array}$ & p value \\
\hline Prognostic variables & & \\
Histologic grade & 0.440 & $<0.0001$ \\
Lymphovascular space invasion & 0.492 & $<0.0001$ \\
Cervical involvement & 0.530 & $<0.0001$ \\
Adnexal involvement & 0.650 & $<0.0001$ \\
Positive cytology & 0.158 & 0.186 \\
Lymph node involvement & 0.201 & 0.234 \\
\hline *Spearman's correlation & &
\end{tabular}

Table 4. The Association of Tumor-free Distance from Serosa and Prognostic Variables

\begin{tabular}{|c|c|c|}
\hline Tumo & $\begin{array}{c}\text {-free distance } \\
\text { r/rho value }\end{array}$ & $\begin{array}{l}\text { om serosa } \\
\text { p value }\end{array}$ \\
\hline \multicolumn{3}{|l|}{ Prognostic variables } \\
\hline Histologic grade & -0.446 & $0.000 *$ \\
\hline Lymphovascular space invasion & -0.494 & $0.000 *$ \\
\hline Cervical involvement & -0.470 & $0.000 *$ \\
\hline Adnexal involvement & -0.344 & $0.004 *$ \\
\hline Positive cytology & -0.292 & $0.015^{*}$ \\
\hline Lymph node involvement & -0.165 & $0.175 * *$ \\
\hline Surgical stage & -0.468 & $0.000 *$ \\
\hline
\end{tabular}

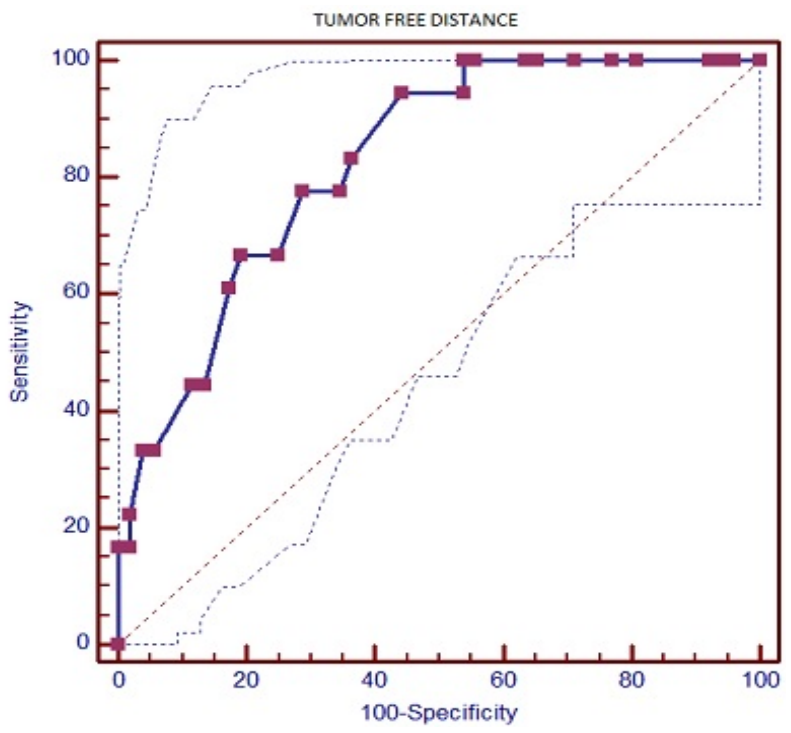

Figure 1. ROC Curve mean-median tumor invasion depth value was $7 \mathrm{~mm}(0-$ $32 \mathrm{~mm}$ ), mean-median tumor free myometrial thickness value was $10 \mathrm{~mm}(0-25 \mathrm{~mm})$. For the predetermination of LVSI, the cut-off value with the highest sensitivity and specificity crossing the ROC curve was calculated to be $11 \mathrm{~mm}$ for tumor free myometrial invasion (Figure 1).

To sum up, all three parameters which were more than 1/2 MI, DOI and TFD were found to have a predictive value for tumor grade, LVSI and cervical involvement. Additionally, the TFD was also found to be significant for predicting adnexal involvement and more than $1 / 2$ MI was found to be significant for predicting nodal metastasis.

\section{Discussion}

Surgical staging is the initial treatment approach in endometrial cancer, including total abdominal hysterectomy, bilaterally salphingo-oopherectomy, abdominal washing, and pelvic and para-aortic lymphadenectomy (Wright et al., 2012). The positive impact of total lymphadenectomy on recurrence was not found in low risk groups (endometrioid type, grade $1-2$, less than $1 / 2$ myometrial invasion, less than $2 \mathrm{~cm}$ tumor diameter) (Bertelsen et al., 2011; Dinkelspiel et al., 2013). When considering the risk/benefit ratio, total lymphadenectomy is not recommended in low risk groups in which the likelihood of lymphatic involvement is low (Sorosky, 2012). Current approach is that the DOI is determined intraoperatively in grade 1-2 tumors and at the presence of more than $1 / 2 \mathrm{MI}$, pelvic and para-aortic lymphadenectomy should be performed (Dinkelspiel et al., 2013; Jaishuen et al, 2014).

Preoperative imaging techniques and immunohistochemical evaluations are insufficient in the detection of nodal involvement (Sorosky, 2012). The DOI and histological grade are the most important predictors of the determination of LN involvement (Cetinkaya et al., 2014; Vargas et al., 2014). It is suggested that not only the DOI but also the tumor distance to the serosa may be prognostic (Schwab et al., 2009; Chattopadhyay et al., 2012). TFD has been found to be effective in detecting LVSI, histological grade and LN metastasis in patients with endometrial cancer (Schwab et al., 2009). Even, TFD is declared to be more valuable as an independent predictive factor than the depth and the rate of myometrial invasion in determination of $\mathrm{LN}$ involvement (Chattopadhyay et al., 2012). However there is no consensus regarding this. In our study, the LN metastasis were found to be more prevalent in the presence of more than $1 / 2 \mathrm{MI}$. The DOI and TFD were not found to be statistically significant for detecting LN metastasis.

Some studies suggest that the TFD is the most effective prognostic factor for detecting recurrence and death of the disease (Chattopadhyay et al., 2012), others assert that the DOI is more effective (Schwab et al., 2009; Geels et al., 2013). DOI was presented with multivariate analysis to be the most effective parameters for determining of LVSI and cervical involvement also (Kondalsamy-Chennaksavan et al., 2010).

Several studies have reported that the cut-off value of the TFD in patients with endometrial cancer which 


\section{Ozlem Ozbilen et al}

demonstrated the best predictive performance value is between 1.75 and $10 \mathrm{~mm}$ (Lee et al., 2009; KondalsamyChennaksavan et al., 2010). In our study, the TFD was measured lower than $11 \mathrm{~mm}$, in all patients with LN metastasis, which was also lower than the cut-off values recommended in published studies (Schwab et al., 2009; Kondalsamy-Chennaksavan et al., 2010; Chattopadhyay et al., 2012). A statistically significant result could not be obtained because LN metastasis was found in just five of seventy patients.

All three parameters analyzed in the current study, which were more than $1 / 2 \mathrm{MI}$, DOI and TFD, found to have a predictive value for tumor grade, LVSI and cervical involvement. Additionally, the TFD was also found to be significant for predicting adnexal involvement. The presence of more than $1 / 2 \mathrm{MI}$ was found to be superior for determining of nodal metastasis. The TFD and the DOI were insufficient for determining nodal metastasis. The shortcoming of this study is that it is retrospective, and the evaluation requires larger prospective studies to put forward predictive cut-off measures of outcome.

In conclusion, our results indicate that, as TFD has a predictive value for tumor grade, LVSI, cervical involvement and also adnexal involvement. It can also contribute to the evaluation of MI by frozen section, in cases with difficulty assessing MI. We need further prospective well-designed studies to clarify the impact of these parameters.

\section{References}

Bertelsen K, Ortoft G, Hansen E (2011). Survival of Danish patients with endometrial cancer in the intermediate-risk group not given postoperative radiotherapy: the Danish Endometrial Cancer Study (DEMCA). Int J Gynecol Cancer, 2,1191-9.

Cetinkaya K, Atalay F, Bacinoglu A (2014). Risk factors of lymph node metastasis with endometrial carcinoma. Asian Pac J Cancer Prev, 15, 6353-6.

Chattopadhyay S, Galaal K, Patel A, et al (2012). Tumor-free distance from serosa is a beter prognostic indicator than depth of invasion and percentage myometrial invasion in endometrioid endometrial cancer. BJOG, 119, 1162-70.

Dinkelspiel HE, Wright JD, Lewin SN, Herzog TJ (2013). Contemporary clinical management of endometrial cancer. Obstet Gynecol Int, 2013, 583891.

Ferlay J, Shin HR, Bray F, et al (2010). Estimates of worldwide burden of cancer in 2008: GLOBOCAN 2008. Int J Cancer, 127, 2893-917.

Geels YP, Pijnenborg JM, van den Berg-van Erp SH, et al (2013). Absolute depth of myometrial invasion in endometrial cancer is superior to the currently used cut-off value of $50 \%$. Gynecol Oncol, 129, 285-91.

Jaishuen A, Kunakornporamat K, Viriyapak B, et al (2014). Incidence and clinical outcomes of non-endometrioid carcinoma of endometrium: Siriraj Hospital experience. Asian Pac J Cancer Prev, 15, 2905-9.

Kondalsamy-Chennaksavan S, van Vugt S, Sanday K, et al (2010). Evaluation of tumor-free distance and depth of myometrial invasion as prognostic factors for lymph node metastasis in endometrial cancer. Int J Gynecol Cancer, 20, 1217-21.

Lee KB, Kim KD, Lee JM, et al (2009). The risk of lymph node metastasis based on myometrial invasion and tumor grade in endometrioid uterine cancers: a multicenter, retrospective Korean study. Ann Surg Oncol, 16, 2882-7.

Quick CM, May T, Horowitz NS, Nucci MR (2012). Low-grade, low-stage endometrioid endometrial adenocarcinoma: a clinicopathologic analysis of 324 cases focusing on frequency and pattern of myoinvasion. Int J Gynecol Pathol, 31, 337-43.

Rahatli S, Dizdar O, Kucukoztas N, et al (2014). Good outcomes of patients with stage IB endometrial cancer with surgery alone. Asian Pac J Cancer Prev, 15, 3891-3.

Schwab KV, O’Malley DM, Fowler JM, Copeland LJ, Cohn DE (2009). Prospective evaluation of prognostic significance of tumor-free distance from uterine serosa in surgically staged endometrial adenocarcinoma. Gynecol Oncol, 112, 146-9.

Siegel R, Naishadham D, Jemal A (2013). Cancer statistics. CA Cancer J Clin, 63, 11-30.

Sorosky JI (2012). Endometrial cancer. Obstet Gynecol, 120, 383-97.

Vargas R, Rauh-Hain JA, Clemmer J, et al (2014). Tumor size, depth of invasion, and histologic grade as prognostic factors of lymph node involvement in endometrial cancer: a SEER analysis. Gynecol Oncol, 133, 216-20.

Wright JD, Barrena Medel NI, Sehouli J, Fujiwara K, Herzog TJ (2012). Contemporary management of endometrial cancer. Lancet, 379, 1352-60. 